\title{
Stokes-Correlometry Analysis of Biological Tissues with Polycrystalline Structure
}

\author{
Alexander Ushenko, Anton Sdobnov, Alexander Dubolazov, Marta Gritsuk, Yurii Ushenko, \\ Alexander Bykov, and Igor Meglinski*, Senior Member, IEEE
}

\begin{abstract}
Utilizing Stokes-correlometry analysis a new diagnostic approach has been introduced for quantitative assessment of polarization images of histological sections of optically anisotropic biological tissues with different morphological structures and physiological conditions. The developed approach is based on the quantitative assessment of coordinate and phase distributions of the Stokes vector of scattered light. A combined use of statistic, correlation and fractal analysis is used for resolving variations in optical anisotropy of biological samples. The proposed combined application of the statistical, correlation and fractal-based evaluates of spatial distributions of 'single-point' polarization azimuth, ellipticity and 'two-point' Stokes vector parameters of polarization images of biological tissues histological sections demonstrates a high accuracy $(A c \geq 90 \%)$ in monitoring of optical anisotropy variations within biological tissues.
\end{abstract}

Index Terms - Polarimetric imaging, polarization, ellipsometry and polarimetry, Stokes-correlometry.

\section{INTRODUCTION}

$\mathrm{T}$ HE optically-based polarimetry is popular experimental approach widely used for non-invasive diagnosis of various turbid tissue-like scattering media [1-6], including those containing optically anisotropic layers [7-9] and fluids [10-12]. With the recent instrumentation developments in the field, a high sensitivity of laser polarimetry in screening of turbid cancerous biological tissues has been achieved [13-16]. The further development of this approach is based on the statistical analysis of averaged values of the polarization parameters (e.g. such as polarization azimuth and ellipticity), obtained for a wide range of variations in the selected samples

Manuscript received XX xxxxx; revised XX xxxxx; accepted XX xxxxx. Date of publication XX xxxxx; date of current version 31.03.2018. This work was partially supported by the Academy of Finland (grant projects: 311698 and 290596); Tomsk State University Academic D. I. Mendeleev Fund Program and the National Research Nuclear University MEPhI's Academic Excellence Project (Contract No. 02.a03.21.0005). Asterisk indicates corresponding author.

A.G. Ushenko, A.V. Dubolazov, M. Gritsuk and Yu.A. Ushenko are with the Chernivtsi National University, 2 Kotsiubynskyi St., Chernivtsi, Ukraine.A.Yu. Sdobnov, A. Bykov with the Opto-Electronic and Measurement Techniques, University of Oulu, P.O. Box 4500, Oulu, 90014, Finland.

* I. Meglinski with the Opto-Electronic and Measurement Techniques, University of Oulu, P.O. Box 4500, Oulu, 90014, Finland; Interdisciplinary Laboratory of Biophotonics, National Research Tomsk State University, Tomsk, 634050, Russia; National Research Nuclear University "MEPhI", Institute of Engineering Physics for Biomedicine (PhysBio), 115409 Moscow, Russia (Correspondence: e-mail: igor.meglinski@oulu.fi and tel.: +358294488888 ). of biological tissues from the series of microscopy images [3$5,17]$. The major application in frame of these studies has been focused on the diagnosis of the biological tissues at different stage of cancer as well as pre-cancerous human tissues with fibrillar structure of polycrystalline networks. Pathological malformations in such tissues are typically accompanied by a significant changes in the phase anisotropy and/or birefringence properties due to the spatial transformation of optical axes of the protein fibrils $[4,18,19]$. It has been demonstrated that statistical analysis of 'one-point' spatial distributions of the polarization azimuth and ellipticity values, obtained from microscopy images of optically thin tissues, as well as the analysis of polycrystalline layers of biological fluids, are quite effective when an assessment of pathological progression is required [10-12,18,19]. The disadvantage of this approach is the lack of data corresponding to the spatial consistency (degree of correlation) of the polarization parameters at different points in the phase-inhomogeneous object field of biological layers. The theoretical basis for obtaining such data is presented in Ref. [20]. Here, a new analytical parameter - the complex degree of mutual polarization (CDMP) is introduced to characterize the correlation structure of polarization-inhomogeneous fields. CDMP characterizes the correlation consistency degree of polarization states (azimuth and ellipticity) at various points of the optical field. This theoretical polarization-correlation approach has been generalized in frames of the polycrystalline structure analysis. Further, this method has been successfully experimentally tested for differentiation of pathological changes in their optical anisotropy [21-26]. Also, the generalization of the CDMP-analysis of polarizationinhomogeneous object fields of biological layers using the "two-point" formalism of the Stokes vector has been theoretically introduced in [27,28]. However, a number of unsolved problems remain in the field of polarization correlometry:

i. the absence of a unified experimental methodology for the parameters distributions mapping on the basis of the synthesis of traditional Stokes-Polarimetry and Stokes-correlometry;

ii. the possibility of diagnostics of weak changes in optical anisotropy of a more wider (comparing to the fibrillar) morphological class of biological tissues.

The importance of these problems solving is due to the fact that in reality, the most of human organs, including liver, stomach, pancreas, spleen, and lungs, have a parenchymal structure, when optical anisotropy are formed by spatially separated within the tissue localized areas. The polarization 
changes associated with birefringence for such optically heterogeneous structure are very small. Therefore, the application of mentioned above statistical approach for such tissue samples is less effective. Thus, further developments of optical polarimetry for tissue diagnosis focused on screening of the parenchymatous tissues and quantitative assessment of the optical anisotropy changes associated with malformations of tissues are expected.

In current paper we present the fundamentals behind of the laser Stokes-polarimetry approach and consider its application, utilizing polarization-correlation mapping of microscopy images of the histological sections of parenchymatous biological tissues, determining the spatial distribution of 'twopoint' Stokes vector parameters.

\section{THEORY OF THE METHOD}

The following assumptions are based on the theoretical model of the Stokes-correlometry method of polarizationinhomogeneous object fields of biological tissues layers:

1. To describe the correlation structure of the stationary distributions of the fields of complex amplitudes of laser light converted by optically anisotropic biological layers, the following mutual spectral density matrix is used [27,28]:

$W_{i, j}\left(r_{1}, r_{2}\right)=E_{i}^{*}\left(r_{1}\right) \cdot E_{j}\left(r_{2}\right), i, j=x, y$.

Here, $r_{1}$ and $r_{2}$ are the coordinates of the neighboring points in the laser radiation field.

Using this matrix operator the following expression for the "two-point" Stokes vector parameters can be obtained:

$$
\begin{aligned}
& S_{1}=W_{x x}\left(r_{1}, r_{2}\right)+W_{y y}\left(r_{1}, r_{2}\right), \\
& S_{2}=W_{x x}\left(r_{1}, r_{2}\right)-W_{y y}\left(r_{1}, r_{2}\right), \\
& S_{3}=W_{x y}\left(r_{1}, r_{2}\right)-W_{y x}\left(r_{1}, r_{2}\right), \\
& S_{4}=i\left[W_{y x}\left(r_{1}, r_{2}\right)+W_{x y}\left(r_{1}, r_{2}\right)\right]
\end{aligned}
$$

where,

$$
\left\{\begin{array}{l}
W_{x x}\left(r_{1}, r_{2}\right)=E_{x}^{*}\left(r_{1}\right) E_{x}\left(r_{2}\right) ; \\
W_{y y}\left(r_{1}, r_{2}\right)=E_{y}^{*}\left(r_{1}\right) E_{y}\left(r_{2}\right) ; \\
W_{x y}\left(r_{1}, r_{2}\right)=E_{x}^{*}\left(r_{1}\right) E_{y}\left(r_{2}\right) ; \\
W_{y x}\left(r_{1}, r_{2}\right)=E_{y}^{*}\left(r_{1}\right) E_{x}\left(r_{2}\right) .
\end{array}\right.
$$

Using relations (2)-(6) the following equations for "twopoint" Stokes vector parameters (hereinafter Stokescorrelometry parameters - SCP) calculation can be obtained:

$$
\begin{aligned}
& S_{1}\left(r_{1}, r_{2}\right)=E_{x}^{*}\left(r_{1}\right) E_{x}\left(r_{2}\right)+E_{y}^{*}\left(r_{1}\right) E_{y}\left(r_{2}\right) ; \\
& S_{2}\left(r_{1}, r_{2}\right)=E_{x}^{*}\left(r_{1}\right) E_{x}\left(r_{2}\right)-E_{y}^{*}\left(r_{1}\right) E_{y}\left(r_{2}\right) ; \\
& S_{3}\left(r_{1}, r_{2}\right)=E_{x}^{*}\left(r_{1}\right) E_{y}\left(r_{2}\right)+E_{y}^{*}\left(r_{1}\right) E_{x}\left(r_{2}\right) ; \\
& S_{4}\left(r_{1}, r_{2}\right)=i\left[E_{y}^{*}\left(r_{1}\right) E_{x}\left(r_{2}\right)-E_{x}^{*}\left(r_{1}\right) E_{y}\left(r_{2}\right)\right] .
\end{aligned}
$$

2. Let us consider the object field of an optically anisotropic biological layer. The complex amplitudes $E(r)$ of each point $r$ of such a field are described by Jones vector $[4,26]$ :

$$
\begin{aligned}
& E(r)=\left(\begin{array}{l}
E_{x} \\
E_{y}
\end{array}\right)(r)=\left(\begin{array}{l}
\left|E_{x}\right| \\
\left|E_{y}\right| \exp \left(\delta_{y}-\delta_{x}\right)
\end{array}\right)(r)= \\
& =\left(\begin{array}{l}
1 \\
\operatorname{tg} \rho \exp (\delta)
\end{array}\right)(r)=\left(\begin{array}{l}
1 \\
\operatorname{tg} \rho(\cos \delta+i \sin \delta)
\end{array}\right)(r)
\end{aligned}
$$

Here, $\operatorname{tg} \rho(r)=\frac{\left|E_{y}\right|(r)}{\left|E_{x}\right|(r)}$ and $\delta(r)=\left(\delta_{y}-\delta_{x}\right)(r)$ is the phase shift between the orthogonal components $\left(\left|E_{x}\right|(r),\left|E_{y}\right|(r)\right)$ of laser wave amplitude.

3. Taking into account (11) the equations (7) - (10) can be rewritten as:

$$
\begin{gathered}
S_{1}\left(r_{1}, r_{2}\right)=\left(1+\operatorname{tg} \rho_{1} \operatorname{tg} \rho_{2} \cos \left(\delta_{1}-\delta_{2}\right)\right)-i\left(\operatorname{tg} \rho_{1} \operatorname{tg} \rho_{2} \sin \left(\delta_{1}-\delta_{2}\right)\right) \\
S_{2}\left(r_{1}, r_{2}\right)=\left(1-\operatorname{tg} \rho_{1} \operatorname{tg} \rho_{2} \cos \left(\delta_{1}-\delta_{2}\right)\right)+i\left(\operatorname{tg} \rho_{1} \operatorname{tg} \rho_{2} \sin \left(\delta_{1}-\delta_{2}\right)\right) \\
S_{3}\left(r_{1}, r_{2}\right)=\left(\operatorname{tg} \rho_{2} \cos \delta_{2}+\operatorname{tg} \rho_{1} \cos \delta_{1}\right)+i\left(\operatorname{tg} \rho_{2} \sin \delta_{2}-\operatorname{tg} \rho_{1} \sin \delta_{1}\right) \\
S_{4}\left(r_{1}, r_{2}\right)=\left(\operatorname{tg} \rho_{1} \sin \delta_{1}+\operatorname{tg} \rho_{2} \sin \delta_{2}\right)+i\left(\operatorname{tg} \rho_{1} \cos \delta_{1}-\operatorname{tg} \rho_{2} \cos \delta_{2}\right)
\end{gathered}
$$

4. Based on (12) - (15), the expressions for the experimentally measured components of the complex parameters of the 'two-point' parameters of the Stokes vector the modulus $S_{i=1 ; 2 ; 3 ; 4}\left(r_{1}, r_{2}\right)$ and phase $\operatorname{Arg} S_{i=1 ; 2 ; 3 ; 4}$ $\left|S_{i=1 ; 2 ; 3 ; 4}\right|$ have been defined as:

$$
\begin{aligned}
& \left\{\begin{array}{l}
\left\{S_{1} \mid\left(r_{1}, r_{2}\right)=\left(\left(1+\operatorname{tg} \rho_{1} \operatorname{tg} \rho_{2} \cos \left(\delta_{1}-\delta_{2}\right)\right)^{2}+\left(\operatorname{tg} \rho_{1} \operatorname{tg} \rho_{2} \sin \left(\delta_{1}-\delta_{2}\right)\right)^{2}\right)^{0.5} ;\right. \\
\operatorname{Arg} S_{2}\left(r_{1}, r_{2}\right)=\operatorname{arctg}\left(\frac{\operatorname{tg} \rho_{1} \operatorname{tg} \rho_{2} \sin \left(\delta_{1}-\delta_{2}\right)}{\left(1+\operatorname{tg} \rho_{1} \operatorname{tg} \rho_{2} \cos \left(\delta_{1}-\delta_{2}\right)\right)}\right) ;
\end{array}\right. \\
& \left\{\begin{array}{l}
\left|S_{2}\right|\left(r_{1}, r_{2}\right)=\left(\left(1-\operatorname{tg} \rho_{1} \operatorname{tg} \rho_{2} \cos \left(\delta_{1}-\delta_{2}\right)\right)^{2}+\left(\operatorname{tg} \rho_{1} \operatorname{tg} \rho_{2} \sin \left(\delta_{1}-\delta_{2}\right)\right)^{2}\right)^{0.5} ; \\
\operatorname{Arg} S_{2}\left(r_{1}, r_{2}\right)=\operatorname{arctg}\left(\frac{\operatorname{tg} \rho_{1} \operatorname{tg} \rho_{2} \sin \left(\delta_{1}-\delta_{2}\right)}{\left(1-\operatorname{tg} \rho_{1} \operatorname{tg} \rho_{2} \cos \left(\delta_{1}-\delta_{2}\right)\right)}\right) ;
\end{array}\right. \\
& \left\{\begin{array}{l}
\left|S_{3}\right|\left(r_{1}, r_{2}\right)=\left(\left(\operatorname{tg} \rho_{2} \cos \delta_{2}+\operatorname{tg} \rho_{1} \cos \delta_{1}\right)^{2}+\left(\operatorname{tg} \rho_{2} \sin \delta_{2}-\operatorname{tg} \rho_{1} \sin \delta_{1}\right)^{2}\right)^{0.5} ; \\
\operatorname{Arg} S_{3}\left(r_{1}, r_{2}\right)=\operatorname{arctg}\left(\frac{\left(\operatorname{tg} \rho_{2} \sin \delta_{2}-\operatorname{tg} \rho_{1} \sin \delta_{1}\right)}{\left(\operatorname{tg} \rho_{2} \cos \delta_{2}+\operatorname{tg} \rho_{1} \cos \delta_{1}\right)}\right) ;
\end{array}\right. \\
& \left\{\begin{array}{l}
\left|S_{4}\right|\left(r_{1}, r_{2}\right)=\left(\left(\operatorname{tg} \rho_{1} \sin \delta_{1}+\operatorname{tg} \rho_{2} \sin \delta_{2}\right)^{2}+\left(\operatorname{tg} \rho_{1} \cos \delta_{1}-\operatorname{tg} \rho_{2} \cos \delta_{2}\right)^{2}\right)^{0.5} ; \\
\operatorname{Arg} S_{4}\left(r_{1}, r_{2}\right)=\operatorname{arctg}\left(\frac{\left(\operatorname{tg} \rho_{1} \cos \delta_{1}-\operatorname{tg} \rho_{2} \cos \delta_{2}\right)}{\left(\operatorname{tg} \rho_{1} \sin \delta_{1}+\operatorname{tg} \rho_{2} \sin \delta_{2}\right)}\right) ;
\end{array}\right.
\end{aligned}
$$

5. Further, for simplification (without affecting the completeness of analysis) relations (16)-(19) can be considered in the approximation of weak phase modulation $\left(\delta_{i} \leq 0,12 \mathrm{rad} ; \cos \delta_{i} \rightarrow 1 ; \sin \delta_{i} \rightarrow \delta_{i}\right)$. Important to note that this assumption is not artificial for optically thin histological sections of rat kidney and liver. It can be shown that for laser light with the wavelength $\lambda=0.63 \mu \mathrm{m}$ within 
geometric thickness $l=30 \mu m$ of completely optically anisotropic layer in biological tissue $\left(\Delta n \approx 10^{-4} \div 1.5 \times 10^{-3}\right.$ [28]) the maximal phase shift ( $\delta_{12} \equiv \delta=\frac{2 \pi}{\lambda} \Delta n l$ ) takes values $0.03 \mathrm{rad} \leq \delta \leq 0.45 \mathrm{rad}$. Moreover, among the birefringent networks or "islets" (clusters of spatially non-oriented protein molecules) there are variations of transverse dimensions of birefringent $(2 \mu m \leq \Delta l \leq 20 \mu m)$ structures, which forms weak phase modulations $(0.002 \mathrm{rad} \leq \Delta \delta \leq 0.3 \mathrm{rad})$ in the polarization-inhomogeneous image plane. Under these conditions Eqs.(16) - (19) can be modified as:

$$
\begin{aligned}
& \left\{\begin{array}{l}
\left|S_{1}\right|\left(r_{1}, r_{2}\right)=\left(\left(1+\operatorname{tg} \rho_{1} \operatorname{tg} \rho_{2}\right)^{2}+\left(\operatorname{tg} \rho_{1} \operatorname{tg} \rho_{2}\left(\delta_{1}-\delta_{2}\right)\right)^{2}\right)^{0.5} ; \\
\operatorname{Arg} S_{2}\left(r_{1}, r_{2}\right)=\operatorname{arctg}\left(\frac{\operatorname{tg} \rho_{1} \operatorname{tg} \rho_{2}\left(\delta_{1}-\delta_{2}\right)}{\left(1+\operatorname{tg} \rho_{1} \operatorname{tg} \rho_{2}\right)}\right) ;
\end{array}\right. \\
& \left\{\begin{array}{l}
\left|S_{2}\right|\left(r_{1}, r_{2}\right)=\left(\left(1-\operatorname{tg} \rho_{1} \operatorname{tg} \rho_{2}\right)^{2}+\left(\operatorname{tg} \rho_{1} \operatorname{tg} \rho_{2}\left(\delta_{1}-\delta_{2}\right)\right)^{2}\right)^{0.5} ; \\
\operatorname{Arg} S_{2}\left(r_{1}, r_{2}\right)=\operatorname{arctg}\left(\frac{\operatorname{tg} \rho_{1} \operatorname{tg} \rho_{2}\left(\delta_{1}-\delta_{2}\right)}{\left(1-\operatorname{tg} \rho_{1} \operatorname{tg} \rho_{2}\right)}\right) ;
\end{array}\right. \\
& \left\{\begin{array}{l}
\left.\left|S_{3}\right|\left(r_{1}, r_{2}\right)=\left(\operatorname{tg} \rho_{2}+\operatorname{tg} \rho_{1}\right)^{2}+\left(\delta_{2} \operatorname{tg} \rho_{2}-\delta_{1} \operatorname{tg} \rho_{1}\right)^{2}\right)^{0.5} ; \\
\operatorname{Arg} S_{3}\left(r_{1}, r_{2}\right)=\operatorname{arctg}\left(\frac{\left(\delta_{2} \operatorname{tg} \rho_{2}-\delta_{1} \operatorname{tg} \rho_{1}\right)}{\left(\operatorname{tg} \rho_{2}+\operatorname{tg} \rho_{1}\right)}\right) ;
\end{array}\right. \\
& \left\{\begin{array}{l}
\left|S_{4}\right|\left(r_{1}, r_{2}\right)=\left(\left(\delta_{1} \operatorname{tg} \rho_{1}+\delta_{2} \operatorname{tg} \rho_{2}\right)^{2}+\left(\operatorname{tg} \rho_{1}-\operatorname{tg} \rho_{2}\right)^{2}\right)^{0.5} ; \\
\operatorname{Arg} S_{4}\left(r_{1}, r_{2}\right)=\operatorname{arctg}\left(\frac{\left(\operatorname{tg} \rho_{1}-\operatorname{tg} \rho_{2}\right)}{\left(\delta_{1} \operatorname{tg} \rho_{1}+\delta_{2} \operatorname{tg} \rho_{2}\right)}\right) .
\end{array}\right.
\end{aligned}
$$

The analysis of the obtained relations $(20)-(23)$ revealed:

i. in case of weak phase fluctuations $\delta_{1 ; 2}$ the value $\left|S_{1-4}\right|\left(r_{1}, r_{2}\right)$ are predominantly determined by the components which are functionally depends on the parameters $\rho_{1 ; 2}$;

ii. the value $\operatorname{Arg} S_{1-4}\left(r_{1}, r_{2}\right)$ has a complex dependence on $\delta_{1 ; 2}$ and $\rho_{1 ; 2}$ in the points of the object field of the optically anisotropic biological layer.

6. The detailed analytical and experimental analysis of the polarimetry of the 3rd and the 4th 'two-point' Stokes vector parameters can be potentiality is used in further experiments. This assumption is based on the fact that the first Stokesvector parameter $S_{1}(r)$ characterizes the full intensity at the point $r$, while the second $S_{2}(r)$ and the third $S_{3}(r)$ parameters are responsible for the characterization of the changes in polarization azimuth and ellipticity. The fourth Stokes-vector parameter $S_{4}(r)$ characterizes the value of polarization ellipticity.

Let us define the characteristic values and ranges of the values of the 3rd and the 4th parameters of the two-point Stokes vector, as well as their modulus and phase. As an object of study, we consider an optically anisotropic biological layer. It forms the value of the phase shift $\delta(r)$ and the ratio $\rho(r)$ between the orthogonal components of the amplitude
$\left|E_{x}\right|(r),\left|E_{y}\right|(r)$. Let us consider (without reducing the completeness of the analysis) that such a layer is illuminated by the linearly polarized beam with azimuth $-0^{0}\left(E_{0}=\left(\begin{array}{l}1 \\ 0\end{array}\right)\right.$.

Table I presents the characteristic values of the "two-point" parameters $S_{3 ; 4}\left(r_{1}, r_{2}\right)$ (relations (7) - (15)) and components of the Stokes vector $\left|S_{4}\right|\left(r_{1}, r_{2}\right)$ and $\operatorname{Arg} S_{3 ; 4}\left(r_{1}, r_{2}\right)$ (relations (17) - (19)) for different polarization states at the points $r, r_{2}$ of the object field of such a layer.

The data given in Table 1 determines the range of variation of the parameters $\left|S_{3 ; 4}\right|\left(r_{1}, r_{2}\right)$ and $\operatorname{ArgS}_{3 ; 4}\left(r_{1}, r_{2}\right)$, depending on the change in the coordinate structure $\left(r_{1}, r_{2}\right)$ of the polarization states $\left(\rho_{1 ; 2}, \delta_{1 ; 2}\right)$ of the object field of the optically anisotropic layer:

$$
\begin{gathered}
0 \leq\left|S_{3 ; 4}\right|\left(r_{1}, r_{2}\right) \leq 1 ; \\
0 \leq \operatorname{ArgS}_{3 ; 4}\left(r_{1}, r_{2}\right) \leq 0.5 \pi .
\end{gathered}
$$

Further, the coordinate $(\Delta r \equiv \Delta x ; \Delta y)$ distributions $\left|S_{i=3 ; 4}(\Delta x ; \Delta y)\right|$ will be called SCP "orientation maps", and $\operatorname{Arg}\left(S_{i=3 ; 4}(\Delta x ; \Delta y)\right)$ - the SCP phase maps.

The described assumptions will be used for the analysis of experimental data of the Stokes-correlometry method of object fields of histological sections of real biological tissues.

\section{Method AND MATERIALS}

Measurements of the coordinate distributions of the values of parameters $\quad\left|S_{i=3}(\Delta x ; \Delta y)\right|, \quad \operatorname{Arg}\left(S_{i=3}(\Delta x ; \Delta y)\right)$, $\left.\mid S_{i=4}(\Delta x ; \Delta y)\right)$, and $\operatorname{Arg}\left(S_{i=4}(\Delta x ; \Delta y)\right)$ were carried out in the experimental arrangement of Stokes-polarimeter. Fig.1 demonstrates the optical scheme of the Sokes-polarimeter $[4,18,19]$.

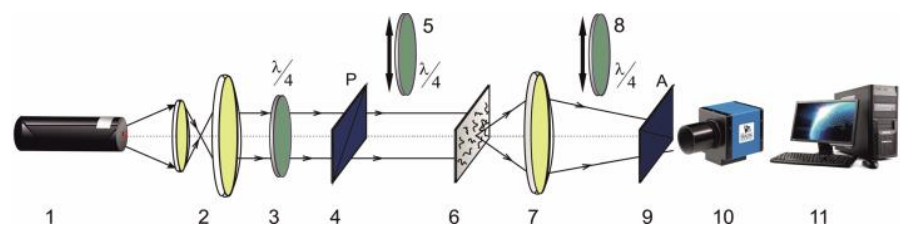

Fig. 1. Optical scheme of polarimeter, where: 1 - He-Ne laser; 2 - collimator; 3 - stationary quarter-wave plate; 5,8 - mechanically movable quarter-wave plates; 4, 9 - polarizer and analyzer; 6 - biological layer; 7 - polarizing microobjective; 10 - CCD camera; 11 - PC.

Biological tissue sample were illuminated by parallel $\left(\varnothing=2 \times 10^{3} \mu \mathrm{m}\right)$ low-intensity (W=5.0 MW) He-Ne laser $(\lambda=0,6328 \mu \mathrm{m}$, Laser, Edmund Optics, USA). Polarization light-source consists of quarter-wave plate (Achromatic True Zero-Order Waveplate (APAW $15 \mathrm{~mm}$, Astropribor, Ukraine) and polarizer $(\mathrm{B}+\mathrm{W}$ XS-Pro Polarizer MRC Nano, Kaesemann, Germany). 
TABLE I.

Characteristic values of the modulus $\left|S_{4}\right|\left(r_{1}, r_{2}\right)$ and phase $\operatorname{Arg} S_{3 ; 4}\left(r_{1}, r_{2}\right)$ of the "two-point" parameters $S_{3 ; 4}\left(r_{1}, r_{2}\right)$ of the STOKES VECTOR OF THE POLARIZATION-INHOMOGENEOUS OBJECT FIELD OF AN OPTICALLY ANISOTROPIC BIOLOGICAL LAYER

\begin{tabular}{|c|c|c|c|c|c|c|c|}
\hline $\begin{array}{l}\rho_{1}, \text { rad; } \\
\delta_{1}, \text { rad }\end{array}$ & $E\left(r_{1}\right)$ & $\begin{array}{l}\rho_{2}, \text { rad } \\
\delta_{2}, \text { rad }\end{array}$ & $E\left(r_{2}\right)$ & $\left|S_{3}\right|\left(r_{1}, r_{2}\right) \mid$ & $\operatorname{ArgS}_{3}\left(r_{1}, r_{2}\right)$ & $\left|S_{4}\right|\left(r_{1}, r_{2}\right) \mid$ & $\operatorname{ArgS}_{4}\left(r_{1}, r_{2}\right)$ \\
\hline \multicolumn{8}{|c|}{ "Collinear" states of polarization in points $r_{1}, r_{2}$} \\
\hline $\begin{array}{l}\rho_{1}=0 \\
\delta_{1}=0\end{array}$ & $\left(\begin{array}{l}1 \\
0\end{array}\right)$ & $\begin{array}{l}\rho_{2}=0 \\
\delta_{2}=0\end{array}$ & $\left(\begin{array}{l}1 \\
0\end{array}\right)$ & 0 & "-" & 0 & "-" \\
\hline $\begin{array}{l}\rho_{1}=0.25 \pi ; \\
\delta_{1}=0\end{array}$ & $\frac{1}{\sqrt{2}}\left(\begin{array}{l}1 \\
1\end{array}\right)$ & $\begin{array}{l}\rho_{2}=0.25 \pi \\
\delta_{2}=0\end{array}$ & $\frac{1}{\sqrt{2}}\left(\begin{array}{l}1 \\
1\end{array}\right)$ & 1 & 0 & 0 & "-" \\
\hline $\begin{array}{l}\rho_{1}=0.25 \pi ; \\
\delta_{1}=0.5 \pi\end{array}$ & $\frac{1}{\sqrt{2}}\left(\begin{array}{l}1 \\
i\end{array}\right)$ & $\begin{array}{l}\rho_{2}=0.25 \pi \\
\delta_{2}=0.5 \pi\end{array}$ & $\frac{1}{\sqrt{2}}\left(\begin{array}{l}1 \\
i\end{array}\right)$ & 1 & 0 & 1 & 0 \\
\hline \multicolumn{8}{|c|}{ "Intermediate" states of polarization in points $r_{1}, r_{2}$} \\
\hline $\begin{array}{l}\rho_{1}=0 \\
\delta_{1}=0\end{array}$ & $\left(\begin{array}{l}1 \\
0\end{array}\right)$ & $\begin{array}{l}\rho_{2}=0.25 \pi \\
\delta_{2}=0\end{array}$ & $\frac{1}{\sqrt{2}}\left(\begin{array}{l}1 \\
1\end{array}\right)$ & $\frac{1}{\sqrt{2}}$ & 0 & $\frac{1}{\sqrt{2}}$ & $0.5 \pi$ \\
\hline $\begin{array}{l}\rho_{1}=0.5 \pi \\
\delta_{1}=0\end{array}$ & $\left(\begin{array}{l}1 \\
0\end{array}\right)$ & $\begin{array}{l}\rho_{2}=0.25 \pi \\
\delta_{2}=0\end{array}$ & $\frac{1}{\sqrt{2}}\left(\begin{array}{l}1 \\
1\end{array}\right)$ & $\frac{1}{\sqrt{2}}$ & 0 & $\frac{1}{\sqrt{2}}$ & $0.5 \pi$ \\
\hline $\begin{array}{l}\rho_{1}=0 \\
\delta_{1}=0\end{array}$ & $\left(\begin{array}{l}1 \\
0\end{array}\right)$ & $\begin{array}{l}\rho_{2}=0.25 \pi \\
\delta_{2}=0.5 \pi\end{array}$ & $\frac{1}{\sqrt{2}}\left(\begin{array}{l}1 \\
i\end{array}\right)$ & $\frac{1}{\sqrt{2}}$ & 0 & $\frac{1}{\sqrt{2}}$ & $0.5 \pi$ \\
\hline
\end{tabular}

"Orthogonal" states of polarization in points $r_{1}, r_{2}$

\begin{tabular}{l|c|l|l|l|l|l|l}
\hline $\begin{array}{l}\rho_{1}=0 ; \\
\delta_{1}=0\end{array}$ & $\left(\begin{array}{l}1 \\
0\end{array}\right)$ & $\begin{array}{l}\rho_{2}=0.5 \pi ; \\
\delta_{2}=0\end{array}$ & $\left(\begin{array}{l}0 \\
1\end{array}\right)$ & 1 & 0 & $0.5 \pi$ \\
\hline $\begin{array}{l}\rho_{1}=0.25 \pi ; \\
\delta_{1}=0\end{array}$ & $\frac{1}{\sqrt{2}}\left(\begin{array}{l}1 \\
1\end{array}\right)$ & $\begin{array}{l}\rho_{2}=-0.25 \\
\delta_{2}=0\end{array}$ & $\frac{1}{\sqrt{2}}\left(\begin{array}{l}1 \\
-1\end{array}\right)$ & 0 & $"-"$ & 1 & $0.5 \pi$ \\
\hline $\begin{array}{l}\rho_{1}=0.25 \pi ; \\
\delta_{1}=0.5 \pi\end{array}$ & $\frac{1}{\sqrt{2}}\left(\begin{array}{l}1 \\
i\end{array}\right)$ & $\begin{array}{l}\rho_{2}=0.25 \pi \\
\delta_{2}=-0.5 \pi\end{array}$ & $\frac{1}{\sqrt{2}}\left(\begin{array}{l}1 \\
-i\end{array}\right)$ & 0 & "-" & 0 & "-" \\
\hline
\end{tabular}

Polarization images of biological layer were projected into the plane of the light-sensitive plate $(m \times n=1280 \times 960$ pixels $)$ of CCD-camera (DMK 41AU02.AS, The Imaging Source, Germany) by polarization microobjective (CFI Achromat P, focal length - $30 \mathrm{~mm}$, numerical aperture -0.1 increase $-4 \times$, Nikon, Japan).

Analysis of the images of biological layers 6 was carried out using polarizer and the quarter-wave plate (see Fig.1).

The measuring method for the modulus $\left|\left(S_{i=3 ; 4}(\Delta x, \Delta y)\right)\right|$ and the phase $\operatorname{Arg}\left(S_{i=3 ; 4}(\Delta x, \Delta y)\right)$ of the SCP consists of the following steps:

i. Biological tissue sample is illuminated by circularly polarized laser beam, passed through the quarter-wave plates and polarizer (see Fig.1); ii. The axis of polarizer-analyzer (in the absence of the quarter wavelength plate) is rotated by the angles $\Theta=0^{0}$, $\Theta=90^{\circ}, \Theta=45^{\circ}, \Theta=135^{\circ}$. The intensities of transmitted radiation $I_{0}^{\otimes} ; I_{90}^{\otimes} ; I_{45}^{\otimes} ; I_{135}^{\otimes}$ are measured;

iii. The values of the first, second and third Stokes vector parameters $\quad S_{i=1 ; 2 ; 3}^{\otimes} \quad-\quad S_{1}^{\otimes}=I_{0}^{\otimes}+I_{90}^{\otimes} ; \quad S_{2}^{\otimes}=I_{0}^{\otimes}-I_{90}^{\otimes} ;$ $S_{3}^{\otimes}=I_{45}^{\otimes}-I_{135}^{\otimes}$ are calculated within each pixel of digital camera (see Fig.1).

iv. Quarter-wave plate is placed in front of polarizeranalyzer, which axis of a greatest speed being oriented at the angles $+45^{0}$ and $-45^{0}$ relative to the transmission plane of polarizer. The intensities $I_{\otimes}^{\otimes} ; I_{\oplus}^{\otimes}$ of the transmitted radiation is measured. 
v. 2-dimensional array of values of the fourth Stokes parameter $S_{4}^{\otimes}=I_{\otimes}^{\otimes}-I_{\oplus}^{\otimes}$ is calculated.

vi. $\quad\left|S_{i=3}(\Delta x ; \Delta y)\right|, \operatorname{Arg}\left(S_{i=3}(\Delta x ; \Delta y)\right), \mid S_{i=4}(\Delta x ; \Delta y), \quad$ and $\operatorname{Arg}\left(S_{i=4}(\Delta x ; \Delta y)\right)$ are calculated utilizing the following ratios:

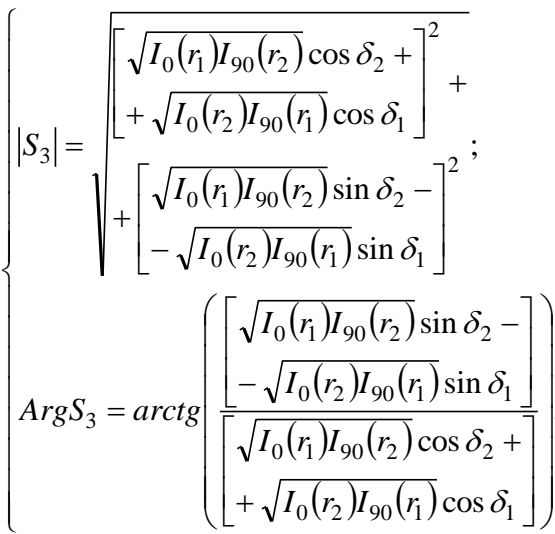

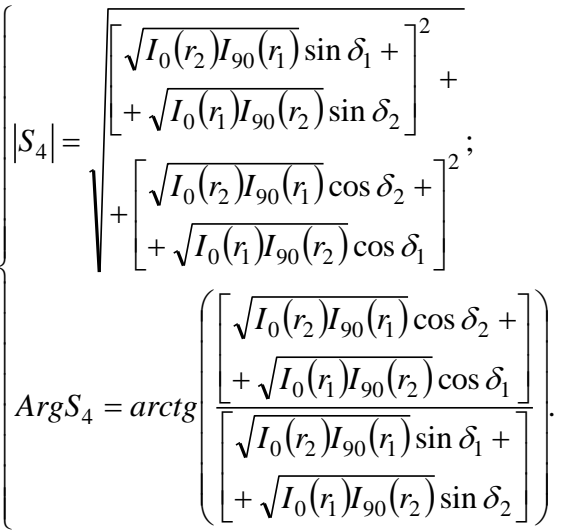

$\delta(r)=\operatorname{arctg}\left[\left(\frac{S_{4}(r) S_{2}(r)}{S_{3}(r)}\right)\left(\frac{1+\frac{I_{90}(r)}{I_{0}(r)}}{1-\frac{I_{90}(r)}{I_{0}(r)}}\right)\right]$.

Here $I_{0}$ and are the intensities of polarizer transmission plane at $0^{0}$ and $90^{\circ}$ orientation; $\delta_{i}$ is the phase shift between the orthogonal components of the laser radiation amplitude in the points with coordinates $r_{1}$ and $r_{2}$.

Optically thin (attenuation coefficient $\tau=0.01$ ) histological sections of biological tissues (thickness $l=25 \mu \mathrm{m} \div 30 \mu \mathrm{m}$ $0.0093 \leq \tau \leq 0.0099)$ taken from two statistically significant (39 samples each) groups of rats healthy and diabetic ones (21 days) were examined:

- kidney tissue - structured tissue with optically anisotropic birefringent collagen network;

- liver tissue - parenchymatous tissue with disordered optically anisotropic protein "islets".

Histological sections of tissue samples were prepared by standard freezing microtome approach.

\section{A. Statistical analysis}

To assess the distribution of random values of $q=\left\{\begin{array}{l}\left|S_{i=3 ; 4}(\Delta x ; \Delta y)\right| \\ \operatorname{Arg}\left(S_{i=3 ; 4}(\Delta x ; \Delta y)\right)\end{array}\right.$ a set of statistical moments of the 1st to 4 th order is calculated $[4,18,19]$.

$$
\begin{aligned}
& Z_{1}=\frac{1}{N} \sum_{i=1}^{N}\left|(q)_{i}\right| ; Z_{2}=\sqrt{\frac{1}{N} \sum_{i=1}^{N}(q)_{i}{ }^{2}} ; \\
& Z_{3}=\frac{1}{Z_{2}{ }^{3}} \frac{1}{N} \sum_{i=1}^{N}(q)_{i}{ }^{3} ; Z_{4}=\frac{1}{Z_{2}{ }^{4}} \frac{1}{N} \sum_{i=1}^{N}(q)_{i}{ }^{4} .
\end{aligned}
$$

Here, $\mathrm{N}$ is the number $(1280 \times 960)$ of pixel at the digital camera.

\section{B. Correlation analysis}

To calculate the average value within all $n$ lines $(1, \ldots, \mathrm{m})$ of the photosensitive plate of CCD-camera (see Fig.1) the autocorrelation function $\overline{\mathrm{K}}(\Delta x)$ with scanning step $\Delta x=1$ pix of distributions of the $q$ parameters values was used $[9,13,17]$ :

$\left.\mathrm{K}_{j=1 \div n}(\Delta x)=\lim _{x \rightarrow 0} \frac{1}{m} \int_{0}^{x}\left[q_{j}^{*}(x=1 \div m)\right] q^{*}(x-\Delta x)\right] d x$.

Here, $\bar{q}$ is the average value of the set $q$.

$\overline{\mathrm{K}}(\Delta x)=\sum_{j=1}^{n} \frac{\mathrm{K}_{j}(\Delta x)}{n}$.

\section{Fractal analysis}

Fractal analysis was performed by calculating the logarithmic dependences $(\log J(q)-\log (v))$ of power spectra of random values $q$ distributions. Here $v=d^{-1}$ is the spatial frequencies defined by the geometrical size $(d)$ of the structural elements of SCS-maps of the polarization-inhomogeneous image of the biological layer. Then, dependences $\log J(q)-\log (v)$ are approximated into curves $\Phi(\eta)$ by least squares method. The classification of the coordinate distributions was performed by the appearance of the obtained curves $[4,18,19]$ :

- distributions of $q$ - fractal at a constant inclination angle $\eta=$ const of the dependence $\Phi(\eta)$ for 2-3 decades of the size change $d$;

- distributions of $q$ - multifractal under the condition of several constant inclination angles $\Phi(\eta)$;

- distributions of $q$ - random under the condition of the absence of stable inclination angles throughout the range of the size change $d$;

The 2-nd order statistical moment has been selected as one of the parameters characterizing the distribution of $\log J(q)-\log (v)$ dependence values: 

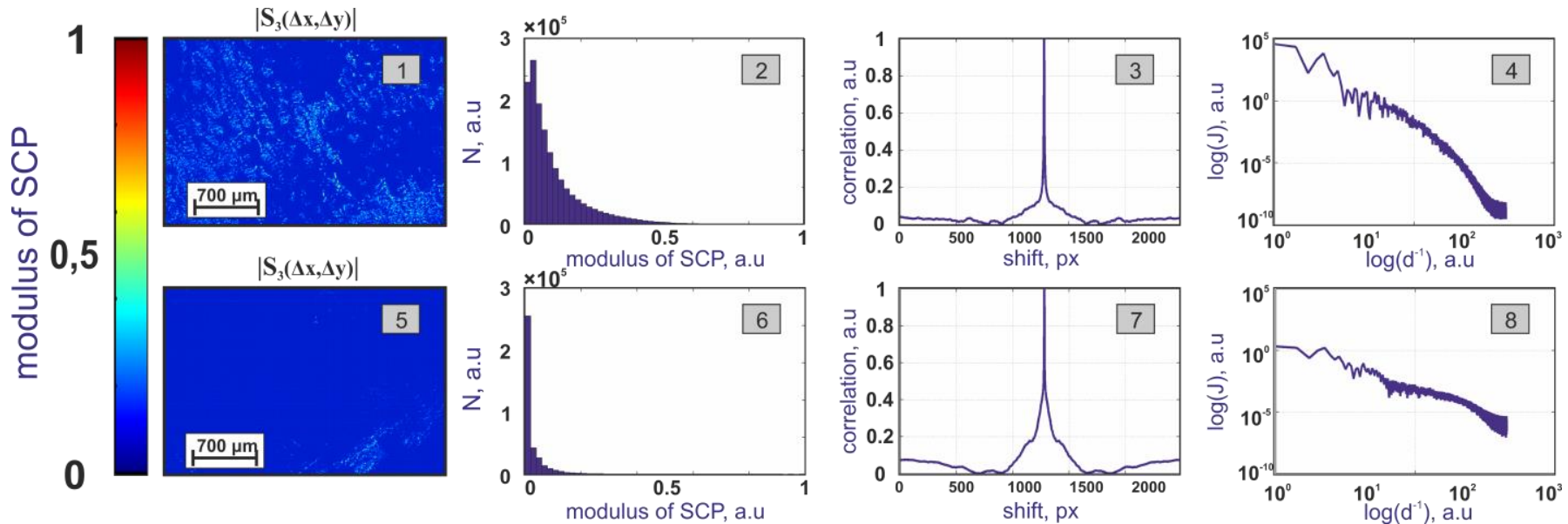

Fig.2. Maps (coordinate distributions (1), (5), histograms (2), (6), autocorrelation functions (3), (7), logarithmic dependences of power spectra (4), (8)) of SCP modulus $\left|S_{i=3}(\Delta x ; \Delta y)\right|$ of polarization-inhomogeneous images of histological sections of a healthy ((1), (2), (3), (4)) and pathologically changed ((5), (6), (7), (8)) rat kidney. See explanations in the text.
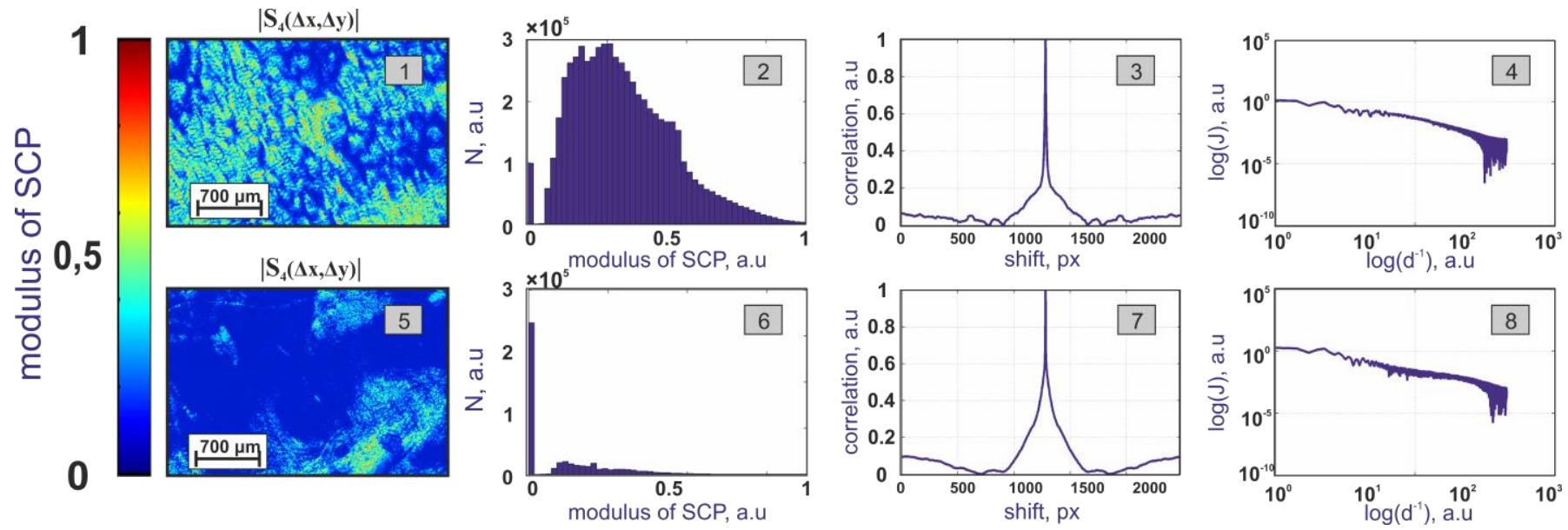

Fig.3. Maps (coordinate distributions (1), (5), histograms (2), (6), autocorrelation functions (3), (7), logarithmic dependences of power spectra (4), (8)) of SCP modulus $\left|S_{i=4}(\Delta x ; \Delta y)\right|$ of polarization-inhomogeneous images of histological sections of a healthy ((1), (2), (3), (4)) and pathologically changed ((5), (6), (7), (8)) rat kidney. See explanations in the text.

$$
D^{f}=\sqrt{\frac{1}{N} \sum_{j=1}^{N}\left(\log J(q)-\log (v)^{2}\right)} .
$$

\section{RESULTS AND DisCUSSION}

\section{A. SCP modulus and phase maps of polarization-}

\section{inhomogeneous images of histological sections of rat kidney}

To compare the theoretical (relations (16)-(23)) and experimental data, irradiation of samples of tissues of internal organs of rats was carried out by a plane-polarized beam with azimuth $0^{0}\left(E_{0}=\left(\begin{array}{l}1 \\ 0\end{array}\right)\right.$.

Fig.2 and Fig.3 present the SCP-maps of the modulus $\left|S_{i=3}(\Delta x ; \Delta y)\right|$ values distribution and $\left|S_{i=4}(\Delta x ; \Delta y)\right|$ microscopic images of histological sections of a healthy and diabetic (21 days) rat kidney respectively.
In a same manner Fig.4 and Fig.5 are shows the SCP-maps of the phase $\operatorname{Arg}\left(S_{i=3}(\Delta x ; \Delta y)\right)$ and $\operatorname{Arg}\left(S_{i=4}(\Delta x ; \Delta y)\right)$ values distribution of microscopic images for healthy and diabetic kidney samples. Statistical analysis of SCP modulus $\left|S_{i=3 ; 4}(\Delta x ; \Delta y)\right|$ maps structure of polarization-inhomogeneous images for kidney samples of both types showed different localization of extremes and half-width of corresponding histograms $N\left(\left|S_{i=3 ; 4}(\Delta x, \Delta y)\right|\right)$ (normal, see Fig.2 and Fig.3, fragments (2) and $N^{*}\left(S_{i=3 ; 4}(\Delta x, \Delta y)\right)$ ), (diabetes, see Fig.2, Fig. 3 fragments (6)). The obtained results can be explained by the following reasons. Due to weak phase modulation, the object field of the histological sections turns out to be sufficiently polarization-homogeneous with respect to the most probable value $E_{0}=\left(\begin{array}{l}1 \\ 0\end{array}\right)$. According to the data presented in Table I, the most probable value in the distributions of the random magnitude of SCP orientation 

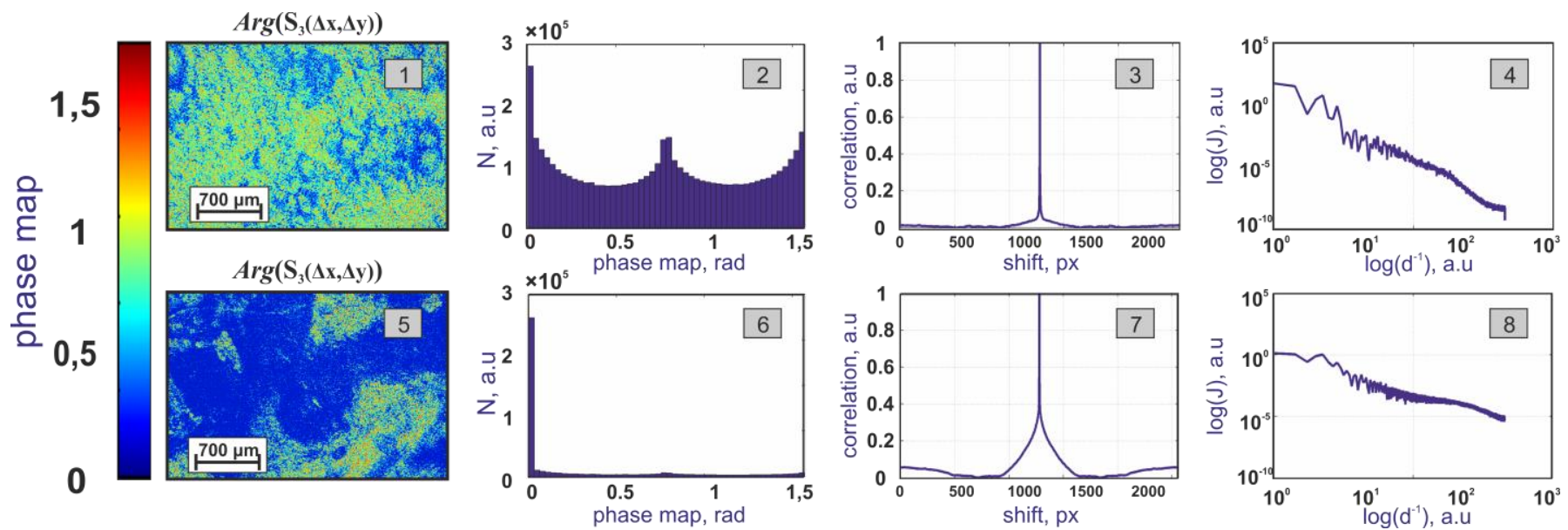

Fig.4. Maps (coordinate distributions (1), (5), histograms (2), (6), autocorrelation functions (3), (7), logarithmic dependences of power spectra (4), (8)) of SCP phase $\operatorname{Arg}\left(S_{i=3}(\Delta x ; \Delta y)\right)$ of polarization-inhomogeneous images of histological sections of a healthy ((1), (2), (3), (4)) and pathologically changed ((5), (6), (7), (8)) rat kidney. See explanations in the text.
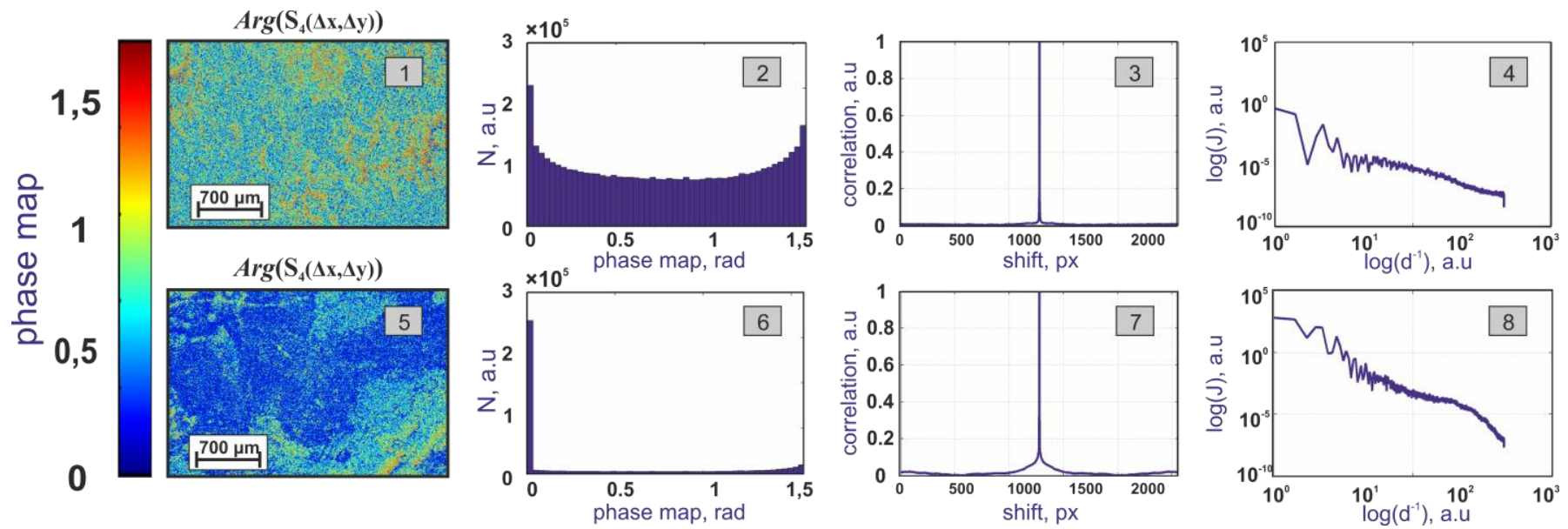

Fig.5. Maps (coordinate distributions (1), (5), histograms (2), (6), autocorrelation functions (3), (7), logarithmic dependences of power spectra (4), (8)) of SCP phase $\operatorname{Arg}\left(S_{i=4}(\Delta x ; \Delta y)\right)$ of polarization-inhomogeneous images of histological sections of a healthy ((1), (2), (3), (4)) and pathologically changed ((4), (5), (7), (8)) rat kidney. See explanations in the text.

maps turns out to be $\left|S_{i=3 ; 4}(\Delta x, \Delta y)\right|=1$. The SCP modulus $\left|S_{i=3 ; 4}(\Delta x, \Delta y)\right|$ values (see Fig.2 and Fig.3, fragments (1), (5)) are primarily defined by the relations $\rho_{1 ; 2}$ between the orthogonal parts of complex amplitudes of neighboring points $r_{1}$ and $r_{2}$ of the histological section plane (equations (22), (23)). In kidney tissue with spatially structured collagen fibrils network differences between the $\rho\left(r_{1}\right)$ and $\rho\left(r_{2}\right)$ are minor $\left(\rho\left(r_{1}\right)-\rho\left(r_{2}\right) \rightarrow 0\right.$ ) for the neighboring points (the scanning step $\Delta r=1$ pix $=2 \mu m$ ). Thus, the following boundary values of modulus $\left|S_{i=3}(\Delta x, \Delta y)\right| \rightarrow 0$ (equation (22)) and $\left|S_{i=3}(\Delta x, \Delta y)\right| \rightarrow 0$ (equation (23)) are quite probable. So, they form the core extremes of histograms $N\left(\left|S_{i=3 ; 4}(\Delta x, \Delta y)\right|\right)$ and $N^{*}\left(\mid S_{i=3 ; 4}(\Delta x, \Delta y)\right)$ (see Fig.2 and Fig.3, fragments (2), (6)). However, for real kidney tissue there is always a certain amount of curvilinear fibrils. For such birefringent networks there is a condition $\rho\left(r_{1}\right)-\rho\left(r_{2}\right) \neq 0$. Thus, the set of other values of SCP modulus $\left|S_{i=3 ; 4}(\Delta x, \Delta y)\right|$ is formed.

Quantitatively, this scenario appears in the redistribution of $\left|S_{i=3 ; 4}(\Delta x, \Delta y)\right| \quad$ values in the entire range $0 \leq\left|S_{i=3 ; 4}(\Delta x, \Delta y)\right| \leq 1$ (see Fig.2 and Fig.3, fragments (2), (6)).

Additionally, the comparative intergroup analysis revealed the correlation coherence increment of polarization states at the points of images of diabetic samples. Analytically it was indicated by the reduction of half-width of histograms $N^{*}\left(\left|S_{i=3 ; 4}(\Delta x ; \Delta y)\right|\right)$ of the modulus $\left|S_{i=3 ; 4}(\Delta x ; \Delta y)\right|$ values distributions of the boundary field of diabetic kidney (see Fig.2 and Fig.3 fragments (6)).

The statistical structure of SCP phase maps is different. The value of $\operatorname{Arg}\left(S_{3 ; 4}(\Delta x, \Delta y)\right)$ (see Fig.4 and Fig.5, fragments (1), (5)) is simultaneously determined by the degree of coordinate coherence of parameters $\rho(r)$ and the value of 
phase shift $\delta(r)$ between the orthogonal components of the amplitude of the laser radiation. It follows from the data analysis of relations that extreme values of $\operatorname{Arg}\left(S_{3}(\Delta x, \Delta y)\right) \rightarrow 0 \quad$ and $\quad \operatorname{Arg}\left(S_{4}(\Delta x, \Delta y)\right) \rightarrow 0.5 \pi \quad$ are determined by fulfillment of these orientation and phase conditions $\quad \Delta r=2 \mu m \rightarrow\left(\begin{array}{l}\rho\left(r_{1}\right) \approx \rho\left(r_{2}\right) ; \\ \delta\left(r_{1}\right) \approx \delta\left(r_{2}\right)\end{array}\right) \quad$ (see $\quad$ Table $\left.\mathrm{I}\right)$. Quantitatively, it is manifested in the prevailing probability of forming the values of histograms $N\left(\operatorname{Arg}\left(S_{3}(\Delta x, \Delta y)\right)=0\right)$ and $N\left(\operatorname{Arg}\left(S_{4}(\Delta x, \Delta y)\right)=0.5 \pi\right)$ (see Fig.4 and Fig.5, fragments (2), (6)). However, this mechanism is not the only one. The phase shift value is also changes because of the difference in size of birefringent collagen fibrils and their curvilinearity.

TABLE II

STATISTICAL, CORRELATION AND FRACTAL PARAMETERS OF SCP MODULUS MAPS OF POLARIZATION-INHOMOGENEOUS IMAGES OF HISTOLOGICAL SECTIONS OF HEALTHY AND PATHOLOGICALLY CHANGED RAT KIDNEY

\begin{tabular}{c|c|c|c|c}
\hline \hline Parameters & \multicolumn{2}{|c|}{$\left|S_{i=3}(\Delta x, \Delta y)\right|$} & \multicolumn{2}{c}{$\left|S_{i=4}(\Delta x, \Delta y)\right|$} \\
\hline Condition & $\begin{array}{l}\text { Norm } \\
(n=39)\end{array}$ & $\begin{array}{l}\text { Diabetes } \\
(n=39)\end{array}$ & $\begin{array}{l}\text { Norm } \\
(n=39)\end{array}$ & $\begin{array}{l}\text { Diabetes } \\
(n=39)\end{array}$ \\
\hline$Z_{1}$ & $0,027 \pm 0,0019$ & $0,016 \pm 0,0011$ & $0,74 \pm 0,034$ & $0,089 \pm 0,004$ \\
\hline$Z_{2}$ & $0,013 \pm 0,0011$ & $0,007 \pm 0,0005$ & $0,23 \pm 0,016$ & $0,081 \pm 0,009$ \\
\hline$Z_{3}$ & $1,35 \pm 0,12$ & $3,78 \pm 0,24$ & $0,69 \pm 0,055$ & $2,07 \pm 0,19$ \\
\hline$Z_{4}$ & $3,24 \pm 0,24$ & $7,18 \pm 0,39$ & $0,82 \pm 0,069$ & $3,95 \pm 0,22$ \\
\hline$Z_{2}^{k}$ & $0,07 \pm 0,005$ & $0,11 \pm 0,009$ & $0,06 \pm 0,005$ & $0,09 \pm 0,007$ \\
\hline$Z_{4}^{k}$ & $2,48 \pm 0,16$ & $0,98 \pm 0,078$ & $1,63 \pm 0,12$ & $0,87 \pm 0,068$ \\
\hline$D^{f}$ & $0,22 \pm 0,017$ & $0,17 \pm 0,013$ & $0,32 \pm 0,021$ & $0,21 \pm 0,017$ \\
\hline \hline
\end{tabular}

So, both of this factors has an influence on the wide range of SCP phase values $\left(0 \leq \operatorname{Arg}\left(S_{i=3 ; 4}(\Delta x, \Delta y)\right) \leq 0.5 \pi\right)$ in polarization-inhomogeneous image of the kidney histological section.

Intergroup analysis showed the decrease of the average and dispersion values characterizing the distributions of SCP phase $\operatorname{Arg}\left(S_{i=3 ; 4}(\Delta x ; \Delta y)\right)$ of polarization-inhomogeneous image of diabetic kidney samples (see Fig.4 and Fig.5 fragments (2), (6)).

From a physical point of view, the changes of the statistical structure of SCP-maps of polarization-inhomogeneous images for the kidney samples of both types can be explained by the fact that the pathological state (diabetes) is accompanied by the destruction of the organ tissue [4,7,18,19,24,26,27]. Optically, this process leads to the destruction of polycrystalline kidney structure while small-scale optically anisotropic structure remains intact. Thus, the polarization image uniformity of these tissues increases. Quantitatively, it is results in the increase of the probability of extreme values of $\left|S_{i=3}(\Delta x ; \Delta y)\right|=0 ; \quad\left|S_{i=4}(\Delta x ; \Delta y)\right|=1 \quad$ and $\operatorname{Arg}\left(S_{i=3}(\Delta x ; \Delta y)\right)=0 ; \quad \operatorname{Arg}\left(S_{i=4}(\Delta x ; \Delta y)\right)=0.5 \pi \quad$ in the distributions of the values of SCP modulus and phase. Therefore, higher order statistical moments that characterize the asymmetry and excess of SCP modulus and phase distributions of the images of the sick kidneys sample are changed by the following scenario

$\left\{\begin{array}{l}\left.Z_{3}\left(\mid S_{i=3 ; 4}(\Delta x ; \Delta y)\right) ; \operatorname{Arg}\left(S_{i=3 ; 4}(\Delta x ; \Delta y)\right)\right) \uparrow ; \\ Z_{4}\left(S_{i=3 ; 4}(\Delta x ; \Delta y) ; \operatorname{Arg}\left(S_{i=3 ; 4}(\Delta x ; \Delta y)\right)\right) \uparrow .\end{array}\right.$

Autocorrelation dependences (Eqs.((30)-(31)) of coordinate distributions of SCP modulus and phase of the pathological kidney images are characterized by the increase of half-width and the decrease in peak sharpness

$$
\left\{\begin{array}{l}
Z_{2}^{K}\left(S_{i=3 ; 4}(\Delta x ; \Delta y) ; \operatorname{Arg}\left(S_{i=3 ; 4}(\Delta x ; \Delta y)\right)\right) \uparrow ; \\
Z_{4}^{K}\left(S_{i=3 ; 4}(\Delta x ; \Delta y) ; \operatorname{Arg}\left(S_{i=3 ; 4}(\Delta x ; \Delta y)\right)\right) \downarrow .
\end{array}\right.
$$

TABLE III

STATISTICAL, CORRELATION AND FRACTAL PARAMETERS OF SCP PHASE MAPS OF POLARIZATION-INHOMOGENEOUS IMAGES OF HISTOLOGICAL SECTIONS OF HEALTHY AND PATHOLOGICALLY CHANGED RAT KIDNEY

\begin{tabular}{c|c|c|c|c}
\hline \hline Parameters & \multicolumn{2}{|c|}{$\operatorname{Arg}\left(S_{i=3}(\Delta x ; \Delta y)\right)$} & \multicolumn{2}{c}{$\operatorname{Arg}\left(S_{i=4}(\Delta x ; \Delta y)\right)$} \\
\hline Condition & $\begin{array}{c}\text { Norm } \\
(n=39)\end{array}$ & $\begin{array}{c}\text { Diabetes } \\
(n=39)\end{array}$ & $\begin{array}{c}\text { Norm } \\
(n=39)\end{array}$ & $\begin{array}{c}\text { Diabetes } \\
(n=39)\end{array}$ \\
\hline$Z_{1}$ & $0,018 \pm 0,0013$ & $0,012 \pm 0,001$ & $0,91 \pm 0,051$ & $0,15 \pm 0,012$ \\
\hline$Z_{2}$ & $0,12 \pm 0,017$ & $0,046 \pm 0,0032$ & $0,14 \pm 0,015$ & $0,074 \pm 0,003$ \\
\hline$Z_{3}$ & $0,64 \pm 0,054$ & $1,95 \pm 0,13$ & $0,56 \pm 0,043$ & $1,79 \pm 0,14$ \\
\hline$Z_{4}$ & $0,78 \pm 0,061$ & $4,09 \pm 0,33$ & $0,67 \pm 0,051$ & $3,29 \pm 0,27$ \\
\hline$Z_{2}^{k}$ & $0,06 \pm 0,005$ & $0,1 \pm 0,009$ & $0,05 \pm 0,005$ & $0,07 \pm 0,007$ \\
\hline$Z_{4}^{k}$ & $3,64 \pm 0,16$ & $1,48 \pm 0,078$ & $4,61 \pm 0,12$ & $2,37 \pm 0,068$ \\
\hline$D^{f}$ & $0,24 \pm 0,017$ & $0,15 \pm 0,013$ & $0,28 \pm 0,021$ & $0,19 \pm 0,017$ \\
\hline \hline
\end{tabular}

It was determined in cases of the Stokes-correlometry mapping that the experimentally measured SCP-maps $\left(\left|S_{i=3 ; 4}(\Delta x, \Delta y)\right|\right.$ and $\left.\operatorname{Arg}\left(S_{i=3 ; 4}(\Delta x ; \Delta y)\right)\right)$ have fractal or multifractal structure. The approximating lines of the logarithmic dependences $\log J\left(\mid S_{i=3 ; 4}(\Delta x, \Delta y)\right)-\log \left(d^{-1}\right)$ and $\log J\left(\operatorname{Arg}\left(S_{i=3 ; 4}(\Delta x, \Delta y)\right)\right)-\log \left(d^{-1}\right)$ are either straight or broken with two inclination angles (see Fig.2 - Fig.5, fragments (4), (8)). It follows from the comparative analysis of these dependences that the distributions of their values are characterized by a specific value of dispersion $D^{f}$ (equation (32)). The smallest one is observed for a polarizationinhomogeneous image of the kidney tissue affected by diabetes. From a physical point of view, it can be related to peculiarity of geometric sizes of the structural elements of SCP-maps which are reduced due to degradation of optical anisotropy of the pathologically changed kidney.

The potentiality of Stokes-correlometry differentiation of the two kidney groups is quantitatively illustrated by the data presented in Table II and Table III.

The procedure for differentiating specimens of histological sections of the kidney tissue consisted of: 

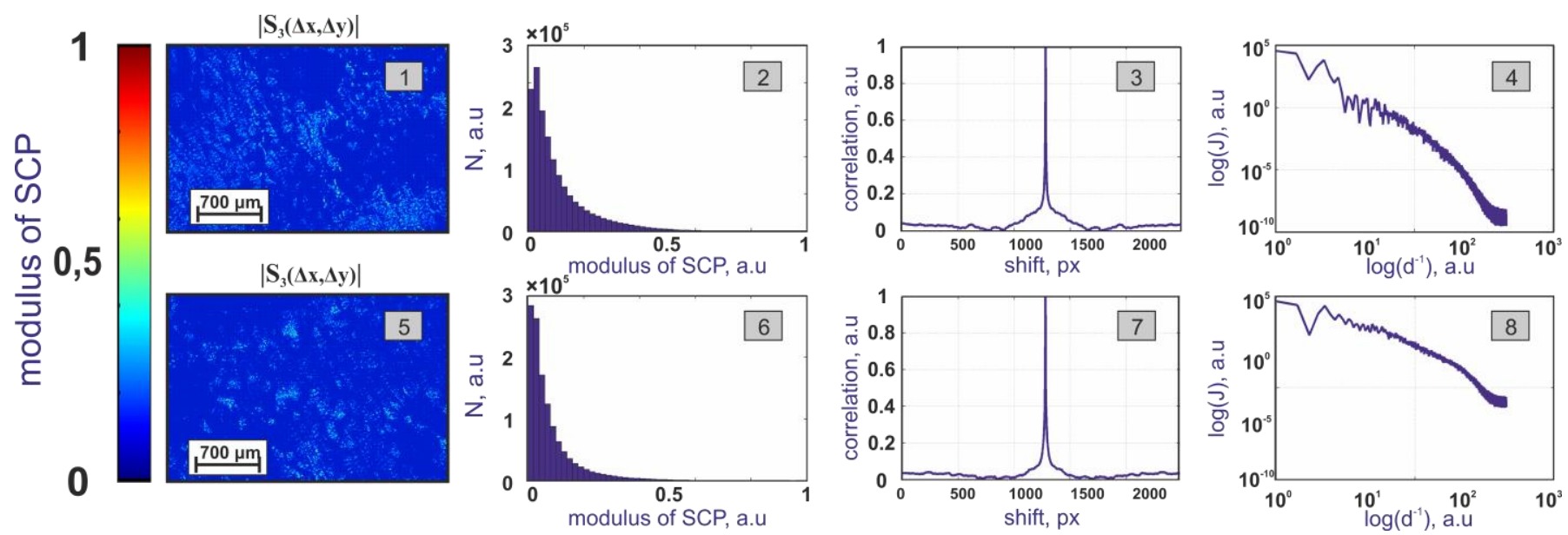

Fig.6. Maps (coordinate distributions (1), (5), histograms (2), (6) autocorrelation functions (3), (7), logarithmic dependences of power spectra (4), (8)) of SCP modulus $\left|S_{i=3}(\Delta x ; \Delta y)\right|$ of polarization-inhomogeneous images of histological sections of a healthy ((1), (2), (3), (4)) and pathologically changed ((5), (6), (7),

(8)) rat liver. See explanations in the text.
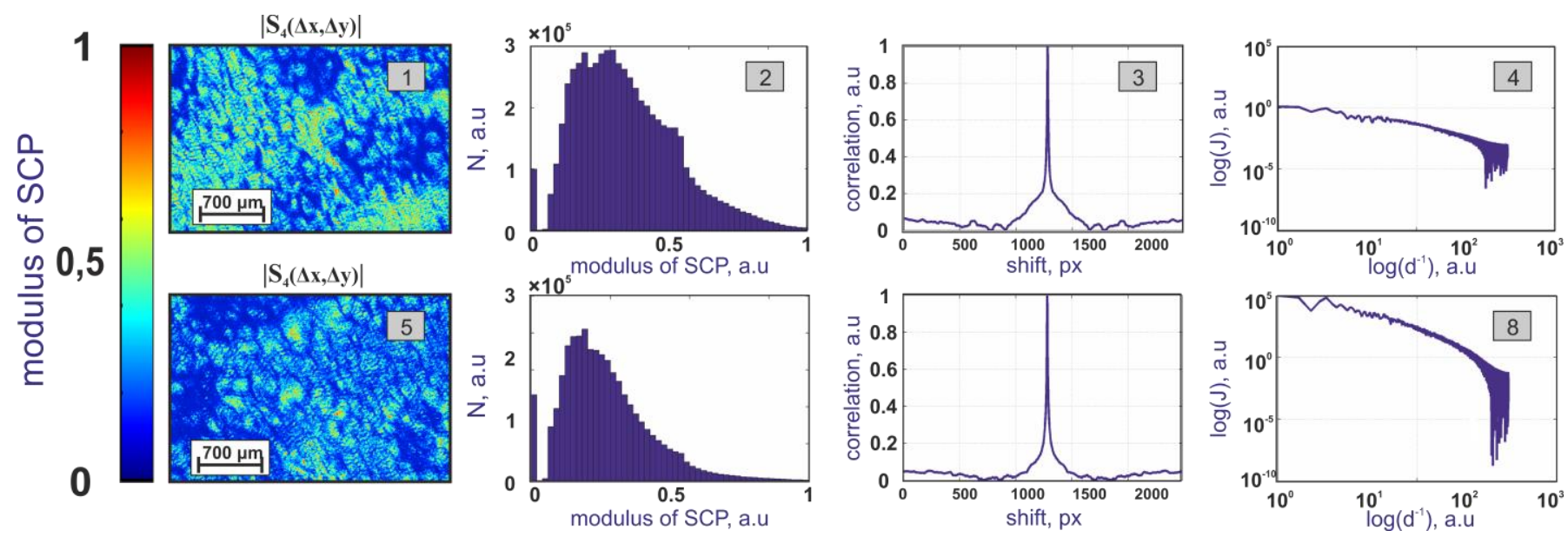

Fig.7. Maps (coordinate distributions (1), (5), histograms (2), (4) autocorrelation functions (3), (7), logarithmic dependences of power spectra (4), (8)) of SCP modulus $\left|S_{i=4}(\Delta x ; \Delta y)\right|$ of polarization-inhomogeneous images of histological sections of a healthy ((1), (2), (5), (6)) and pathologically changed ((3), (4), (7), (8)) rat liver. See explanations in the text.

i. calculation of the set of objective parameters $\mathrm{P}=\left(\begin{array}{l}Z_{i=1 ; 2 ; 3 ; 4} ; \\ Z_{i=2 ; 4}^{k} ; \\ D^{f}\end{array}\right)$ characterizing the distributions of the

modulus $\left|\left(S_{i=3 ; 4}(\Delta x, \Delta y)\right)\right|$ and the phase $\operatorname{Arg}\left(S_{i=3 ; 4}(\Delta x, \Delta y)\right)$ of the SCP;

ii. detection of the maximal differences between the parameters $\mathrm{P}$;

iii. calculation of the mean values $\overline{\mathrm{P}}$ and their errors within a certain partial layer $\pm \sigma$ for each group of samples;

iv. search of the balanced accuracy $A c, \%$ of the diagnostic test on the basis of the following relations determined for each group of samples [30-32]: sensitivity ( $S e=\frac{a}{a+b} 100 \%$ ) specificity $\quad\left(S p=\frac{c}{c+d} 100 \%\right)$ and balanced accuracy
( $A c=\frac{S e+S p}{2}$ ), where $a$ and $b$ are the number of correct and wrong diagnoses within group 2;c and $d$ are the same numbers within group 1

The data analysis revealed the following differences between the set of objective parameters characterizing the maps of SCP-modulus of polarization-inhomogeneous images for the healthy and diabetic kidney samples:

- $\Delta Z_{1}=1.67-8.11$ times; $\Delta Z_{2}=1.85-2.83$ times; $\Delta Z_{3}=2.8-3$ times; $\Delta Z_{4}=2.21-4.81$ times;

- $\Delta Z_{2}^{k}=1.5-1.57$ times; $\Delta Z_{4}^{k}=1.87-2.53$ times;

- $\Delta D^{f}=1.29-1.52$ times.

These data were obtained for the SCP phase distributions:

- $\Delta Z_{1}=1.51-6.06$ times; $\Delta Z_{2}=2.1-2.6$ times; $\Delta Z_{3}$ $=3.04-3.19$ times; $\Delta Z_{4}=4.91-5.24$ times;

- $\Delta Z_{2}^{k}=1.4-1.66$ times; $\Delta Z_{4}^{k}=1.95-2.45$ times; $\Delta D^{f}=1.47-1.58$ times. 
Maps of SCP modulus and phase of polarizationinhomogeneous images of rat liver histological sections

Fig.6 and Fig.7 show the SCP- maps of the distributions of modulus values $\left(S_{i=3}(\Delta x ; \Delta y)\right)$ and $\left(S_{i=4}(\Delta x ; \Delta y)\right)$ of microscopic images of liver parenchymatous tissue of the healthy and diabetic rats (21 days). Whereas, in Fig.8 and Fig.9 SCP- maps of the distributions of phase values $\operatorname{Arg}\left(S_{i=3}(\Delta x ; \Delta y)\right)$ and $\operatorname{Arg}\left(S_{i=4}(\Delta x ; \Delta y)\right)$ of microscopic images for the liver samples of the healthy and diabetic rat (21 days) are presented.

The combined analysis of the structure of SCP modulus $\left|S_{i=3 ; 4}(\Delta x ; \Delta y)\right|$ and phase $\operatorname{Arg}\left(S_{i=3 ; 4}(\Delta x ; \Delta y)\right)$ of polarizationinhomogeneous fields liver samples of both healthy and diabetic types showed the same patterns for optically anisotropic manifestations of diabetes, as those found in the previous studies of the kidney images (see Fig.6 - Fig.9).

TABLE IV

STATISTICAL, CORRELATION AND FRACTAL PARAMETERS OF SCP MODULUS MAPS OF POLARIZATION-INHOMOGENEOUS IMAGES OF HISTOLOGICAL SECTIONS OF HEALTHY AND PATHOLOGICALLY CHANGED RAT LIVER.

\begin{tabular}{c|c|c|c|c}
\hline \hline Parameters & \multicolumn{2}{|c|}{$\left|S_{i=3}(\Delta x ; \Delta y)\right|$} & \multicolumn{2}{c}{$\left|S_{i=4}(\Delta x ; \Delta y)\right|$} \\
\hline Condition & $\begin{array}{c}\text { Norm } \\
(n=39)\end{array}$ & $\begin{array}{c}\text { Diabetes } \\
(n=39)\end{array}$ & $\begin{array}{c}\text { Norm } \\
(n=39)\end{array}$ & $\begin{array}{c}\text { Diabetes } \\
(n=39)\end{array}$ \\
\hline$Z_{1}$ & $0.073 \pm 0,0047$ & $0,058 \pm 0,0032$ & $0,36 \pm 0,023$ & $0,25 \pm 0,014$ \\
\hline$Z_{2}$ & $0,15 \pm 0,012$ & $0,11 \pm 0,005$ & $0,32 \pm 0,016$ & $0,21 \pm 0,013$ \\
\hline$Z_{3}$ & $0,65 \pm 0,047$ & $1,27 \pm 0,062$ & $0,48 \pm 0,025$ & $0,87 \pm 0,051$ \\
\hline$Z_{4}$ & $0,54 \pm 0,034$ & $1,42 \pm 0,079$ & $0,56 \pm 0,039$ & $0,79 \pm 0,037$ \\
\hline$Z_{2}^{k}$ & $0,068 \pm 0,005$ & $0,074 \pm 0,004$ & $0,086 \pm 0,005$ & $0,093 \pm 0,007$ \\
\hline$Z_{4}^{k}$ & $2,58 \pm 0,16$ & $3,88 \pm 0,21$ & $1,13 \pm 0,061$ & $0,78 \pm 0,059$ \\
\hline$D^{f}$ & $0,22 \pm 0,019$ & $0,17 \pm 0,015$ & $0,33 \pm 0,023$ & $0,24 \pm 0,016$ \\
\hline \hline
\end{tabular}

TABLE V

STATISTICAL, CORRELATION AND FRACTAL PARAMETERS OF SCP PHASE MAPS OF POLARIZATION-INHOMOGENEOUS IMAGES OF HISTOLOGICAL SECTIONS OF HEALTHY AND PATHOLOGICALLY CHANGED RAT LIVER

\begin{tabular}{c|c|c|c|c}
\hline \hline Parameters & \multicolumn{2}{|c|}{$\operatorname{Arg}\left(S_{i=3}(\Delta x ; \Delta y)\right)$} & \multicolumn{2}{c}{$\operatorname{Arg}\left(S_{i=4}(\Delta x ; \Delta y)\right)$} \\
\hline Condition & $\begin{array}{c}\text { Norm } \\
(n=39)\end{array}$ & $\begin{array}{c}\text { Diabetes } \\
(n=39)\end{array}$ & $\begin{array}{c}\text { Norm } \\
(n=39)\end{array}$ & $\begin{array}{c}\text { Diabetes } \\
(n=39)\end{array}$ \\
\hline$Z_{1}$ & $0,88 \pm 0,049$ & $0,67 \pm 0,034$ & $0,64 \pm 0,041$ & $0,52 \pm 0,037$ \\
\hline$Z_{2}$ & $1,16 \pm 0,062$ & $0,82 \pm 0,057$ & $1,04 \pm 0,061$ & $0,72 \pm 0,045$ \\
\hline$Z_{3}$ & $0,59 \pm 0,032$ & $0,83 \pm 0,057$ & $0,37 \pm 0,024$ & $0,58 \pm 0,041$ \\
\hline$Z_{4}$ & $0,89 \pm 0,053$ & $1,37 \pm 0,071$ & $0,62 \pm 0,042$ & $0,96 \pm 0,051$ \\
\hline$Z_{2}^{k}$ & $0,051 \pm 0,0031$ & $0,072 \pm 0,006$ & $0,028 \pm 0,003$ & $0,036 \pm 0,005$ \\
\hline$Z_{4}^{k}$ & $2,78 \pm 0,18$ & $1,86 \pm 0,26$ & $3,07 \pm 0,22$ & $4,71 \pm 0,31$ \\
\hline$D^{f}$ & $0,27 \pm 0,013$ & $0,21 \pm 0,019$ & $0,28 \pm 0,017$ & $0,19 \pm 0,013$ \\
\hline \hline
\end{tabular}

The differences between the statistical, correlation and fractal parameters describing the coordinate maps of SCP modulus and the phase of polarization-inhomogeneous images for liver samples are quantitatively illustrated in Table IV and Table V.

The obtained data analysis revealed the following differences between the set of objective parameters characterizing the maps of SCP modulus of polarization- inhomogeneous images of kidney histological sections of the healthy and diabetic rat:

- $\Delta Z_{1}=1.26-1.44$ times; $\Delta Z_{2}=1.36-1.52$ times; $\Delta Z_{3}=1.81-1.95$ times; $\Delta Z_{4}=1.41-2.63$ times;

- $\Delta Z_{2}^{k}=1.05-1.14$ times; $\Delta Z_{4}^{k}=1.44-1.51$ times;

- $\Delta D^{f}=1.29-1.37$ times.

It was obtained for the SCP phase distributions:

- $\Delta Z_{1}=1.23-1.31$ times; $\Delta Z_{2}=1.41-1.44$ times; $\Delta Z_{3}=1.41-1.57$ times; $\Delta Z_{4}=1.54-1.56$ times;

- $\Delta Z_{2}^{k}=1.2-1.41$ times; $\Delta Z_{4}^{k}=1.49-1.53$ times;

- $\Delta D^{f}=1.29-1.47$ times.

The most sensitive data are marked with $*$ in Tables VI and VII.

Comparing the results of statistical, correlation and fractal analyses of the maps of SCP modulus and phase of polarization-inhomogeneous images of the structured (kidney) and parenchymatous (liver) rat tissues, significant sensitivity to the changes in optical anisotropy of the "islet" parenchymatous structures has been found; the values of the objective parameters has a 2 -fold difference.

TABLE VI

ACCURACY OF THE METHODS OF STOKES-CORRELOMETRY AND LASER POLARIMETRY OF RAT KIDNEY FIBRILLAR TISSUE IN THE DIFFERENTIAL DIAGNOSTICS OF DIABETES

\begin{tabular}{c|c|c|c|c|c|c}
\hline \hline Parameters & \multicolumn{6}{|c}{$A c, \%$} \\
\cline { 2 - 7 } & $\left|S_{3}\right|$ & $\left|S_{4}\right|$ & $A r g S_{3}$ & $A r g S_{4}$ & $\alpha(x, y)$ & $\beta(x, y)$ \\
\hline$Z_{1}$ & $90^{*}$ & $92^{*}$ & $96^{*}$ & $97^{*}$ & 65 & 68 \\
\hline$Z_{2}$ & $91^{*}$ & $90^{*}$ & $94^{*}$ & $96^{*}$ & 64 & 67 \\
\hline$Z_{3}$ & $92^{*}$ & 87 & $97^{*}$ & $95^{*}$ & 82 & 83 \\
\hline$Z_{4}$ & $90^{*}$ & $91^{*}$ & $95^{*}$ & $98^{*}$ & 80 & 81 \\
\hline$Z_{2}^{k}$ & 82 & 81 & 85 & 87 & 62 & 64 \\
\hline$Z_{4}^{k}$ & $93^{*}$ & $93^{*}$ & $98^{*}$ & $98^{*}$ & 83 & 82 \\
\hline$D^{f}$ & 80 & 82 & 84 & 86 & 67 & 68 \\
\hline \hline
\end{tabular}

TABLE VII

ACCURACY OF THE METHODS OF STOKES-CORRELOMETRY AND LASER POLARIMETRY OF RAT LIVER TISSUE IN THE DIFFERENTIAL DIAGNOSTICS OF DIABETES

\begin{tabular}{c|c|c|c|c|c|c}
\hline \hline Parameters & \multicolumn{6}{|c}{$A c, \%$} \\
\cline { 2 - 7 } & $\left|S_{3}\right|$ & $\left|S_{4}\right|$ & $\operatorname{ArgS}_{3}$ & $\operatorname{Arg} S_{4}$ & $\alpha(x, y)$ & $\beta(x, y)$ \\
\hline$Z_{1}$ & 85 & 83 & $92^{*}$ & $91^{*}$ & 63 & 65 \\
\hline$Z_{2}$ & 86 & 88 & $90^{*}$ & $92^{*}$ & 61 & 63 \\
\hline$Z_{3}$ & 84 & 82 & $91^{*}$ & $90^{*}$ & 79 & 78 \\
\hline$Z_{4}$ & $90^{*}$ & $90^{*}$ & $90^{*}$ & $92^{*}$ & 77 & 75 \\
\hline$Z_{2}^{k}$ & 78 & 77 & 80 & 81 & 58 & 59 \\
\hline$Z_{4}^{k}$ & $90^{*}$ & $90^{*}$ & $92^{*}$ & $92^{*}$ & 74 & 77 \\
\hline$D^{f}$ & 76 & 75 & 80 & 81 & 60 & 61 \\
\hline \hline
\end{tabular}

To determine the Stokes-correlometry diagnostic efficiency the obtained results were compared with the data obtained by conventional laser polarimetry for the same samples. 

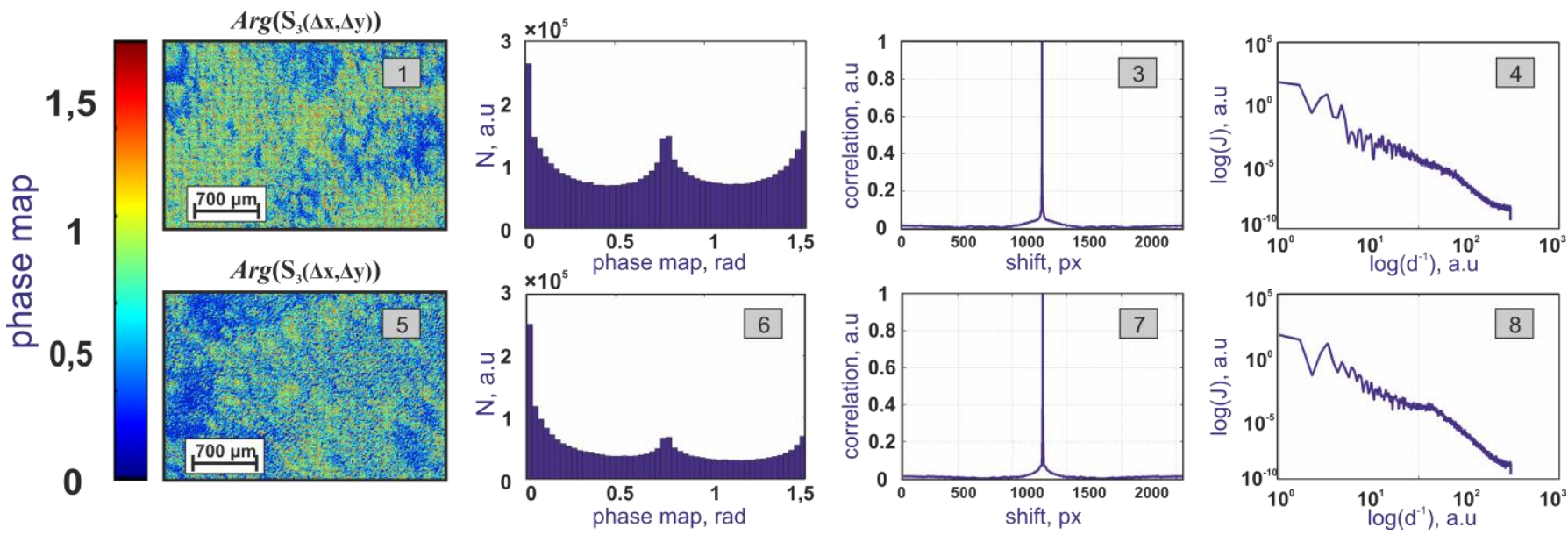

Fig.8. Maps (coordinate distributions (1), (5), histograms (2), (6) autocorrelation functions (3), (7), logarithmic dependences of power spectra (4), (8)) of SCP phase $\operatorname{Arg}\left(S_{i=3}(\Delta x ; \Delta y)\right)$ of polarization-inhomogeneous images of histological sections of a healthy ((1), (2), (3), (4)) and pathologically changed ((5), (6), (7), (8)) rat liver. See explanations in the text.
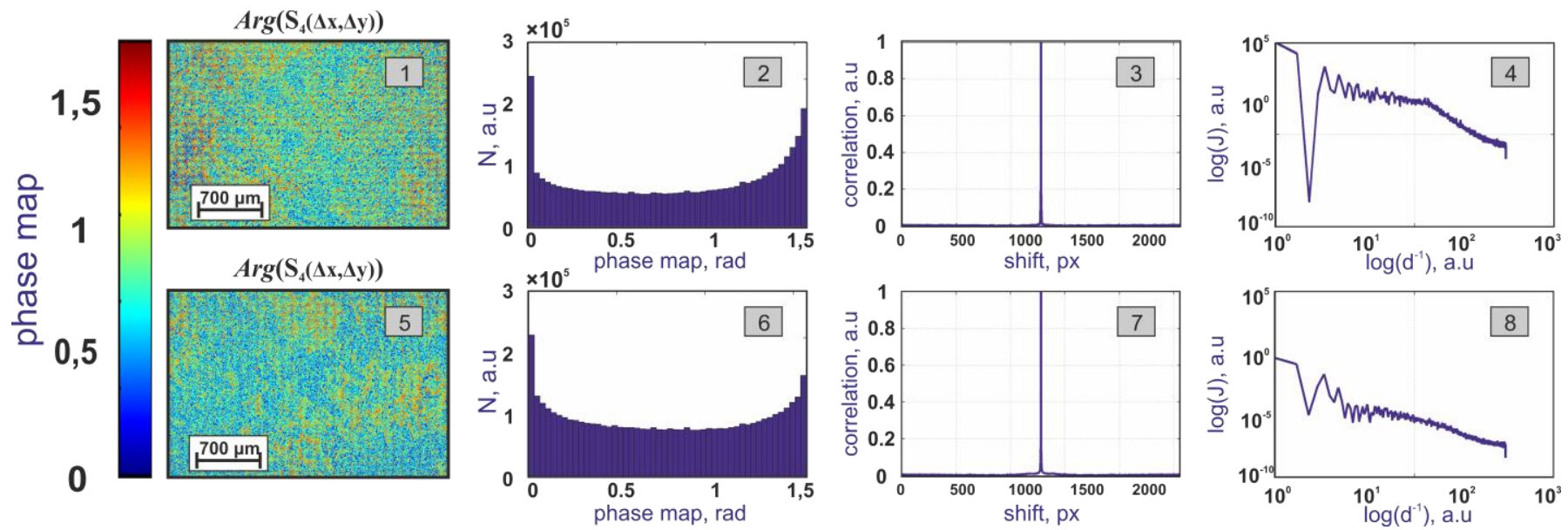

Fig.9. Maps (coordinate distributions (1), (5), histograms (2), (4) autocorrelation functions (3), (7), logarithmic dependences of power spectra (4), (8)) of SCP phase $\operatorname{Arg}\left(S_{i=4}(\Delta x ; \Delta y)\right)$ of polarization-inhomogeneous images of histological sections of a healthy ((1), (2), (5), (6)) and pathologically changed ((3), (4), (7), (8)) rat liver. See explanations in the text.

\section{STOKES-CORRELOMETRY APPROACH}

The Stokes-correlometry method of SCP modulus $\left|S_{i=3 ; 4}(\Delta x ; \Delta y)\right|$ and phase $\operatorname{Arg}\left(S_{i=3 ; 4}(\Delta x ; \Delta y)\right)$ distributions of polarization-inhomogeneous images was compared with the that of polarization mapping of the azimuth and ellipticity values distributions for the corresponding microscopic images of kidney (Table VI) and liver (Table VII) samples (two healthy and two diabetic groups of rats, respectively).

The complex study showed significantly greater accuracy for the methods of Stokes-correlometry in the differentiation of weak changes in optical anisotropy of histological sections of the structured ( $94 \% \leq \max A c \leq 98 \%$ ) and parenchymatous $(90 \% \leq \max A c \leq 92 \%)$ biological tissues of internal organs of healthy and diabetic rats.

\section{CONCLUSIONS}

A new diagnostic approach based on determination of the spatial distributions of the modulus and phase of 'two-point' Stokes-vector parameters of polarization-inhomogeneous images of histological sections of biological tissues with different morphological structures and physiological states has been introduced. The SCP-mapping of microscopy polarization images of two groups (normal and diabetic samples) of biological tissues with the structured collagen birefringent fibrillar networks (kidney), and with the 'islet' isotropic-anisotropic structure (liver) were examined. The combined use of statistical, correlation and fractal analysis of spatial distribution of 'single-point' azimuth and ellipticity and 'two-point' Stokes-vector parameters of polarization images of histological sections demonstrated the high accuracy 
( $A c \geq 90 \%$ ) in diagnosis of optical anisotropy variations within biological tissues.

\section{REFERENCES}

[1] T. Novikova, et al., "Polarized Light for Biomedical Applications", $J$. Biomed. Opt., Vol.21, 071001, 2016.

[2] N.G. Khlebtsov, et al., "Introduction to Light Scattering by Biological Objects: Extinction and Scattering of Light in Disperse Systems", in: Handbook of Optical Biomedical Diagnostics, Ed. V. Tuchin, SPIE Press, (2016), Chapter 1.

[3] T.T. Tower, and R.T. Tranquillo, "Alignment Maps of Tissues: I. Microscopic Elliptical Polarimetry," Biophys. J., vol. 81, pp. 29542963, 2001.

[4] A.G. Ushenko, and V.P. Pishak, "Laser Polarimetry of Biological Tissue: Principles and Applications," in Handbook of Coherent-Domain Optical Methods: Biomedical Diagnostics, Environmental and Material Science, Kluwer Academic Publishers, Vol.1, V.V. Tuchin, Ed., 2004, pp. 93-138.

[5] V.V. Tuchin, L. Wang, and D.A. Zimnyakov, Optical Polarization in Biomedical Applications, New York, USA, 2006.

[6] N. Ghosh, and I.A. Vitkin, "Tissue polarimetry: concepts, challenges, applications and outlook," J. Biomed. Opt., vol. 16, pp. 110801, 2011.

[7] V.A. Ushenko, and M.S. Gavrylyak, "Azimuthally invariant Muellermatrix mapping of biological tissue in differential diagnosis of mechanisms protein molecules networks anisotropy," Proc. SPIE, vol. 8812, pp.88120Y, 2013.

[8] V.O. Ushenko, et al., "Two-dimensional Mueller matrix phase tomography of self-similarity birefringence structure of biological tissues," Proc. SPIE, vol. 8487, pp. 84870W, 2012.

[9] V.A. Ushenko, N. D. Pavlyukovich, and L. Trifonyuk, "SpatialFrequency Azimuthally Stable Cartography of Biological Polycrystalline Networks," In. J. Opt., pp. 683174, 2013.

[10] Yu.A. Ushenko, et al., "Mueller-matrix diagnostics of optical properties of polycrystalline networks of human blood plasma," Opt. Spectros., vol. 112, pp. 884-892, 2012.

[11] Yu.A. Ushenko et al, "Wavelet-analysis of polarization maps of human blood plasma," Opt. Spectros., vol. 113, pp. 332-343, 2012.

[12] V.A. Ushenko, O.V. Dubolazov, and A.O. Karachevtsev, "Two wavelength Mueller matrix reconstruction of blood plasma films polycrystalline structure in diagnostics of breast cancer," Appl. Opt., vol. 53, pp. B128-B139, 2014.

[13] A. Pierangelo, et al., "Ex-vivo characterization of human colon cancer by Mueller polarimetric imaging," Opt. Express, 19, 1582-1593, 2011.

[14] N.K. Das, et al., "Quantitative assessment of submicron scale anisotropy in tissue multifractality by scattering Mueller matrix in the framework of Born approximation", Opt. Communications, Vol.413, pp.172-178, 2018.

[15] N.K. Das, et al., "Submicron scale tissue multifractal anisotropy in polarized laser light scattering", Laser Phys. Lett., Vol.15, No.3, 035601, 2018.

[16] B. Kunnen, et al., "Application of circularly polarized light for noninvasive diagnosis of cancerous tissues and turbid tissue-like scattering media", J. Biophoton., Vol.8, No.4, pp.317 - 323, 2015.

[17] S.L. Jacques, "Polarized light imaging of biological tissues," in: Handbook of Biomedical Optics, CRC Press, Boca Raton, London, New York, D. Boas, C. Pitris, and N. Ramanujam, Eds., 2011, pp. 649-669.

[18] O.V. Angelsky et al., "Statistical, Correlation and Topological Approaches in Diagnostics of the Structure and Physiological State of Birefringent Biological Tissues," in Handbook of Photonics for Biomedical Science, CRC Press, Taylor\&Francis group: Boca Raton, London, New York, V.V. Tuchin, Ed. 2010, pp.283-322.

[19] Y.A. Ushenko, et al., "Diagnostics of Structure and Physiological State of Birefringent Biological Tissues: Statistical, Correlation and Topological Approaches," in Handbook of Coherent-Domain Optical Methods, Springer Science+Business Media New York, 2013, pp.107148.

[20] J. Ellis and A. Dogariu, "Complex degree of mutual polarization," Opt. Lett. vol. 29, pp. 536-538, 2004.

[21] V.P. Prysyazhnyuk et al., "Polarization-dependent laser autofluorescence of the polycrystalline networks of blood plasma films in the task of liver pathology differentiation," Appl. Opt., vol. 55, pp. B126-B132, 2016.
[22] A.V. Dubolazov, et al., "Birefringence images of polycrystalline films of human urine in early diagnostics of kidney pathology," Appl. Opt. vol. 55, pp. B85-B90, 2016

[23] Yu.A. Ushenko, et al., "Spatial-frequency Fourier polarimetry of the complex degree of mutual anisotropy of linear and circular birefringence in the diagnostics of oncological changes in morphological structure of biological tissues," Quantum. Electron., vol. 42, pp. 727-732, 2012.

[24] V.A. Ushenko, et al., "Complex degree of mutual coherence of biological liquids," Proc. SPIE, vol. 8882, pp. 88820V, 2013.

[25] V.A. Ushenko, and M.P. Gorsky, "Complex degree of mutual anisotropy of linear birefringence and optical activity of biological tissues in diagnostics of prostate cancer," Opt. Spectrosc., vol. 115, pp. 290-297, 2013.

[26] Yu.A. Ushenko, et al., "Jones-matrix mapping of complex degree of mutual anisotropy of birefringent protein networks during the differentiation of myocardium necrotic changes," Appl. Opt., vol. 55, pp. B113-B119, 2016.

[27] J. Tervo, T. Setala, and A. Friberg, "Degree of coherence for electromagnetic fields," Opt. Expr., vol. 11, pp. 1137-1143, 2003.

[28] J. Tervo, T. Setala, and A. Friberg, "Two-point Stokes parameters: interpretation and properties," Opt. Lett., vol. 34, pp. 3074-3076, 2009.

[29] V.V. Tuchin, Tissue Optics: Light Scattering Methods and Instruments for Medical Diagnostics, 3rd ed., Vol. PM 254, SPIE Press, Bellingham, Washington, 2015.

[30] L.D. Cassidy, "Basic concepts of statistical analysis for surgical research," J. Surg. Res., vol. 128, pp. 199-206, 2005.

[31] C.S. Davis, Statistical methods of the analysis of repeated measurements, New York: Springer-Verlag, 2002.

[32] A. Petrie and B. Sabin, Medical Statistics at a Glance, Blackwell Publishing, 2005, pp. 157. 